JEEP 2011, 00007 (2011)

DOI: $10.1051 /$ jeep/201100007

(C) Owned by the authors, published by EDP Sciences, 2011

\title{
Calcul thermodynamique des phases du système Fe-N
}

\author{
M. Keddam
}

Laboratoire de Technologie des Matériaux, Département de S.D.M, Faculté de G.M et G.P, U.S.T.H.B, B.P N³2, 16111, El-Alia, Bab-Ezzouar, Alger, Algérie.

Email : keddam@yahoo.fr

\begin{abstract}
Résumé. Les équilibres thermodynamiques des phases, entre les nitrures du fer $\gamma$ ' et $\varepsilon$, ont été étudiés au moyen de deux approches thermodynamiques. La première approche concerne l'application du modèle de la solution régulière ou sous- régulière basé sur le formalisme Calphad. La seconde approche thermodynamique est relative à l'utilisation de la théorie des isothermes d'absorption de l'azote basée sur le modèle de Gosky-Bragg-Williams. En comparant les résultats issus des deux approches thermodynamiques, il a été montré que les résultats de la théorie des isothermes d'absorption concordent plus avec les résultats de l'expérience.
\end{abstract}

\section{Introduction}

Ce travail concerne l'étude des limites de solubilité de l'azote dans les phases du système binaire Fe-N à une température inférieure à $863 \mathrm{~K}$. Ce diagramme d'équilibre est d'une importance cruciale pour l'étude de la nitruration gazeuse du fer pur et des aciers.

En vue de décrire la cinétique de croissance des couches nitrurées, il est nécessaire de connaître les limites de solubilité de l'azote aux interfaces des phases considérées qui peuvent être accessibles par le calcul thermodynamique. Une étude comparative a été menée sur la base de deux approches thermodynamiques.

Le calcul thermodynamique a montré que la théorie des isothermes d'absorption de l'azote donne de meilleurs résultats.

\section{Modèle thermodynamique selon le formalisme Calphad}

L'analyse de la stabilité thermodynamique des phases $\mathrm{du}$ système binaire $\mathrm{Fe}-\mathrm{N}$ requiert l'étude thermodynamique de chaque phase dans son domaine d'existence. Le diagramme d'équilibre binaire Fe-N donne les domaines d'existence des phases à la condition d'équilibre thermodynamique qui minimise l'énergie libre du système considéré.

Plusieurs auteurs ont auparavant étudié le système Fe-N de point de vue thermodynamique, nous pouvons citer Hillert et Staffansson [1], Agren [2], Frisk [3] et Du [4].
Kunze [5] a réévalué le système $\mathrm{Fe}-\mathrm{N}$ en tenant compte des propriétés du fer pur et les valeurs des énergies libres de Gibbs des phases ont été calculées dans le domaine de températures 600 à $2200 \mathrm{~K}$. les auteurs Kei et Wang [6] ont montré par calcul thermodynamique que les phases $\alpha, \gamma^{\prime}$ et $\varepsilon$ sont également stables aux températures inférieures à $623 \mathrm{~K}$. De plus, les auteurs Frisk [3] et Kunze [5] considèrent que la phase $\gamma^{\prime}$ est de stœchiométrie fixe tandis qu'elle est définie par Du et al. [4,7], comme une phase ayant un domaine de composition très étroit, ce qui est proche de la réalité.

Ces diagrammes d'équilibre de phases Fe-N, donnés par ces auteurs, ont été déterminés à partir de l'utilisation du modèle thermodynamique de la solution régulière ou sous régulière appliqué à une solution d'insertion de l'azote dans les phases considérés.

Ce modèle thermodynamique est également appelé modèle des deux sous-réseaux [7]. Le premier sousréseau contient les atomes du fer tandis que le second sous-réseau est rempli partiellement par l'azote qui vient occuper les sites octaédriques. La non idéalité de la solution solide est prise en compte par l'introduction d'une énergie d'excès exprimée sous forme polynomiale. L'énergie d'ordre magnétique est donnée par le modèle proposé par Inden [8].

Le modèle des deux sous-réseaux de Hillert et Staffansson [1] permet donc de donner l'expression de l'énergie libre de Gibbs de chaque phase du système binaire Fe-N.

Pour la formulation chimique correspondante à une mole de la phase solide $\phi:(\mathrm{Fe})_{\mathrm{a}}(\mathrm{N}, \mathrm{Va})_{\mathrm{c}}$, on obtient l'expression suivante 
$G^{\phi}{ }_{m}=y^{\phi}{ }_{N} G_{F e: N}^{\phi, 0}+y_{V a}^{\phi} G_{F e: V a}^{\phi, 0}+c . R \cdot T \cdot\left[y_{N}^{\phi} \ln \left(y_{N}^{\phi}\right)+\right.$

$\left.y_{V a}^{\phi} \ln \left(y_{V a}^{\phi}\right)\right]+G_{m}^{\phi, e x c}+G_{m}^{\phi, m g}$

$G_{m}^{\phi, e x c}=y_{N N}^{\phi} y_{V a}^{\phi} \cdot L_{F e: N, V a}^{\phi}$

avec :

$$
\begin{aligned}
& L_{F e: N, V a}^{\phi}=L_{F e: N, V a}^{0}+L_{F e: N, V a}^{1} \cdot\left(y_{N}-y_{V a}\right) \\
& G_{m}^{\phi, m g}=R \cdot T \cdot \ln \left(1+\beta^{\phi}\right) \cdot f\left(T / T_{C}^{\phi}\right)
\end{aligned}
$$

Le paramètre thermodynamique $L_{F e: N, V a}^{\phi}$ représente le coefficient d'interaction entre le fer, l'azote et la lacune interstitielle (Va). Si $L_{F e: N: V a}^{1}=0$, la solution solide est donc traitée comme une solution régulière. $\mathrm{R}$ est la constante des gaz parfaits et $\mathrm{T}$ la température absolue en Kelvin. La variable $y_{i}^{\phi}$ avec $\mathrm{i}=(\mathrm{N}, \mathrm{Va})$ représente la fraction en sites de l'élément i dans son propre sous - réseau tandis que les paramètres a et $\mathrm{c}$ sont les nombres de sites dans chaque sous- réseau. Les paramètres $G_{F e: i}^{\phi, 0}$ avec $(\mathrm{i}=\mathrm{N}, \mathrm{Va})$, sont données en référence aux enthalpies de l'état le plus stable, a savoir : $(298,15 \mathrm{~K}$ et $0,1 \mathrm{MPa})$.

Le terme $G_{m}^{\phi, e x c}$ décrit la non idéalité de la solution solide et $G_{m}^{\phi, m g}$ est la contribution énergétique due à l'effet magnétique, expression suggéré par Inden [8]. $T_{C}^{\phi}$ est la température de Curie et $\beta^{\phi}$ le magnéton de Bohr.

Pour établir les équilibres thermodynamiques entre les phases, il faut définir les expressions des potentiels chimiques par rapport à l'azote et au fer.

$\mu^{\phi}{ }_{N}=\partial G_{m}^{\phi} / c \cdot \partial y_{N}^{\phi}$

et

$\mu_{F e}^{\phi}=1 / a \cdot\left[G_{m}^{\phi}+y_{N}^{\phi} \cdot \partial G_{m}^{\phi} / \partial y_{V a}^{\phi}\right]$

L'équilibre thermodynamique est atteint à pression et température constantes si l'énergie libre du système est minimale, cela revient à écrire l'égalité des potentiels chimiques dans chaque phase par rapport aux éléments considérés. Par exemple, pour étudier l'équilibre entre les phases $\alpha$ et $\gamma$, on doit résoudre le système d'équations non linéaires suivant par la méthode numérique de Newton-Raphson:

$\mu_{N}^{\alpha}=\mu_{N}^{\gamma}$

et

$\mu_{F e}^{\alpha}=\mu_{F e}^{\gamma}$

Dans le cas où la phase $\gamma^{\prime}$ est stoechiométrique et ayant pour formule chimique $\mathrm{Fe}_{4} \mathrm{~N}$, son potentiel chimique sera donc égal à son énergie libre standard de formation $G_{\mathrm{Fe}_{4} \mathrm{~N}}^{0}$ à une température inférieure à $863 \mathrm{~K}$.

$G_{F e 4 N}^{0}=4 \cdot \mu_{F e}^{\alpha}+\mu_{N}^{\alpha}$

\section{Théorie des isothermes d'absorption de l'azote}

Cette théorie décrit bien la thermodynamique du système binaire $\mathrm{Fe}-\mathrm{N}$ car elle donne des résultats en conformité avec les prévisions du diagramme expérimental Fe-N. Par exemple, les phases $\varepsilon$ et $\gamma^{\prime}$ et présentent des domaines de composition non nuls contrairement aux travaux de Frisk [3] ou Kunze [5] où elles sont considérée comme des phases stœchiométriques ayant des compositions fixes. Le tableau 1 fournit les expressions analytiques, issues de la théorie des isothermes d'absorption d'azote, pour le calcul des compositions des frontières entre différentes phases qui sont : $\left(\alpha / \alpha+\gamma^{\prime}\right),\left(\alpha+\gamma^{\prime} / \gamma^{\prime}\right),\left(\gamma^{\prime} / \varepsilon+\gamma^{\prime}\right)$ et $\left(\varepsilon+\gamma^{\prime} / \varepsilon\right)$.

Dans ces équations, la variation de la composition des frontières de phases s'exprime en terme d'occupation de la fraction en site d'azote, $y_{N}^{\phi}\left(\phi=\alpha, \gamma^{\prime}, \varepsilon\right)$, dans son propre sous-réseau, en fonction de la température. [N] est la fraction atomique de l'azote. $c_{N}^{\phi}$ est la concentration d'azote donnée en $\mathrm{mol} / \mathrm{m}^{3}, K_{N, o}^{\phi}$ étant le potentiel de référence de la phase $\phi$ exprimé en $\quad \mathrm{Pa}^{-1 / 2}$ $\left(1 \mathrm{~Pa}^{-1 / 2}=318,3 \mathrm{~atm}^{-1 / 2}\right), \mathrm{n}$ est le nombre de sites d'azote par site de fer, $N_{A v}$ est le nombre $\mathrm{d}$ 'Avogadro $=6,022 \times 10^{23}$ atomes $/$ mole. Les équations ainsi que les valeurs utiles pour le calcul du potentiel nitrurant de référence $K_{N, 0}^{\phi}$ et n, sont rassemblées dans le tableau 2. Ce tableau donne les expressions mathématiques nécessaires au calcul du potentiel nitrurant de référence, $K_{N, 0}^{\phi}\left(\phi=\alpha, \gamma^{\prime}, \varepsilon\right)$ exprimé en $P a^{-1 / 2}$.

Le tableau 3 regroupe les expressions du potentiel de nitruration aux frontières des phases, $K_{N, \kappa / \omega}(\kappa, \omega=$ $\left.\alpha, \gamma^{\prime}, \varepsilon\right)$ en fonction de l'intervalle de température.

Les données thermodynamiques tirées des tableaux 1,2 et 3 permettent de tracer le diagramme de Lehrer (potentiel nitrurant- inverse de la température) actualisé par Maldzinski [12], selon une échelle semilogarithmique, qui fixe le choix du potentiel nitrurant à utiliser en vue de favoriser l'apparition ou non d'une phase nitrure au détriment d'une autre. 
Tableau 1. Données utiles pour le calcul de la composition aux frontières de phases.

\begin{tabular}{|c|c|c|c|c|}
\hline $\begin{array}{c}\text { Frontières } \\
\text { de phases }\end{array}$ & $\mathrm{T}(\mathrm{K})$ & $\phi$ & $y_{N}^{\phi}\left\{=\frac{[N]}{1-[N]}=N_{A v} \frac{V^{\phi}}{n} c_{N}^{\phi}\right\}$ \\
\hline$\alpha / \alpha+\gamma^{\prime}$ & $623-863$ & $\alpha$ & $\exp \left\{-\frac{4541}{T}-1.32\right\}$ \\
\hline$\alpha+\gamma^{\prime} / \gamma^{\prime}$ & $623-863$ & $\gamma^{\prime}$ & $\frac{1}{4}\left\{1-\exp \left\{-\frac{7558}{T}+2,978+\ln \left[\frac{K_{N, 0}^{\gamma^{\prime}}}{K_{N, \alpha / \gamma^{\prime}}}-\frac{K_{N, \alpha / \gamma^{\prime}}}{K_{N, 0}^{\gamma^{\prime}}}\right]\right\}\right\}$ \\
\hline$\gamma^{\prime} / \varepsilon^{+\gamma^{\prime}}$ & $623-870$ & $\gamma^{\prime}$ & $\frac{1}{4}\left\{1-\exp \left\{-\frac{7558}{T}+2,978+\ln \left[\frac{K_{N, 0}^{\gamma^{\prime}}}{K_{N, \gamma^{\prime} / \varepsilon}}-\frac{K_{N, \gamma^{\prime} \varepsilon}}{K_{N, 0}^{\gamma^{\prime}}}\right]\right\}\right\}$ \\
\hline$\varepsilon^{+\gamma^{\prime} / \varepsilon .}$ & $623-870$ & $\varepsilon$ & $\frac{5,758.10^{-2}+6,621 \times 10^{-4} T-5,345 \times 10^{-7} T^{2}}{}$ \\
& & & $0,94242-6,621 \times 10^{-4} T+5,345 \times 10^{-7} T^{2}$ \\
\hline
\end{tabular}

Tableau 2. Données nécessaires au calcul du potentiel nitrurant de référence

\begin{tabular}{|c|c|c|}
\hline Phase $\phi$ & $\begin{array}{c}\log \left(\begin{array}{c}K_{N, 0}(\phi) \\
N, 0\end{array}\right) \\
\text { en } \mathrm{Pa}^{-1 / 2}\end{array}$ & $\mathrm{n}$ \\
\hline$\alpha$ & $-11,56+\frac{9096}{T}$ & 3 \\
\hline$\gamma^{\prime}$ & $-12,5+\frac{6,35 \times 10^{3}}{T}$ & 1 \\
\hline$\varepsilon$ & $-4,92+\frac{3,59 \times 10^{3}}{T}$ & 1 \\
\hline
\end{tabular}

Le tableau 4 englobe les équations décrivant les isothermes d'absorption des phases du système binaire $\mathrm{Fe}-\mathrm{N}$ en fonction de la fraction en site d'azote, $y_{N}^{\phi}\left(\phi=\alpha, \gamma^{\prime}, \varepsilon\right)$, en relation avec le potentiel nitrurant $K_{N}$. Les données utilisées dans ce tableau sont tirées des références [9-11].

Pour rappel, cette théorie repose sur l'étude la dissociation du mélange gazeux $\left(\mathrm{NH}_{3}-\mathrm{H}_{2}\right)$ au contact des phases $\alpha, \gamma^{\prime}$ et $\varepsilon$ [9-11]. Il est à noter que cette théorie reproduit d'une manière parfaite les résultats obtenus expérimentalement. Les paramètres physiques des modèles issus de cette théorie ont été optimisés au moyen des résultats expérimentaux se trouvant dans le diagramme de Lehrer (Figure 1) actualisé par Maldzinski et al. [12].

Tableau 3. Données utiles pour la construction du diagramme de Lehrer

\begin{tabular}{|c|c|c|}
\hline$\kappa / \omega$ & $\mathrm{T}(\mathrm{K})$ & $\ln r_{N, \kappa / \omega}$ \\
\hline$\alpha / \gamma^{\prime}$ & $573-863$ & $\frac{4555}{T}-12,88$ \\
$\gamma^{\prime} / \varepsilon$ & & $-9,63+$ \\
& & $\sqrt{\frac{60536}{T}-56,85}$ \\
\hline
\end{tabular}

Ce diagramme a été établi en considérant l'équilibre thermodynamique entre le fer et l'atmosphère de réaction. Le mélange gazeux $\mathrm{NH}_{3}-\mathrm{H}_{2}$ ) peut être ajusté par le biais du potentiel nitrurant. Ce paramètre thermodynamique permet de contrôler la nature des phases nitrures et il est exprimé en fonction des pressions partielles de l'hydrogène et de l'ammoniac comme suit :

$$
K_{N}=\frac{p_{N H_{3}}}{p_{H_{2}}{ }^{1,5}}
$$




\begin{tabular}{|c|c|}
\hline Phase $\phi$ & $y_{N}^{\phi}$ \\
\hline$\alpha / \alpha+\gamma^{\prime}$ & $\frac{K_{N}}{K_{N, 0}^{\alpha}}$ \\
\hline$\alpha+\gamma^{\prime} / \gamma^{\prime}$ & $\frac{1}{4}-\frac{1}{4} \exp \left(2,98-\frac{7,56 \times 10^{3}}{T}\right) \cdot\left\{\frac{K_{N, 0}^{\gamma^{\prime}}}{K_{N}}-\frac{K_{N}}{K_{N, 0}^{\gamma^{\prime}}}\right\}$ \\
\hline$\gamma^{\prime} / \varepsilon^{+} \gamma^{\prime}$ & $\left.\frac{1}{2}-65,9 \times 10^{-3} \exp \left(-2,57 \frac{K_{N}}{K_{N, 0}^{\varepsilon}}\right\}-95,3 \times 10^{-3} \exp -26,9 \frac{K_{N}}{K_{N, 0}^{\varepsilon}}\right\}$ \\
\hline
\end{tabular}

Tableau 4. Expressions des isothermes d'absorption en fonction de la fraction en site de l'azote.

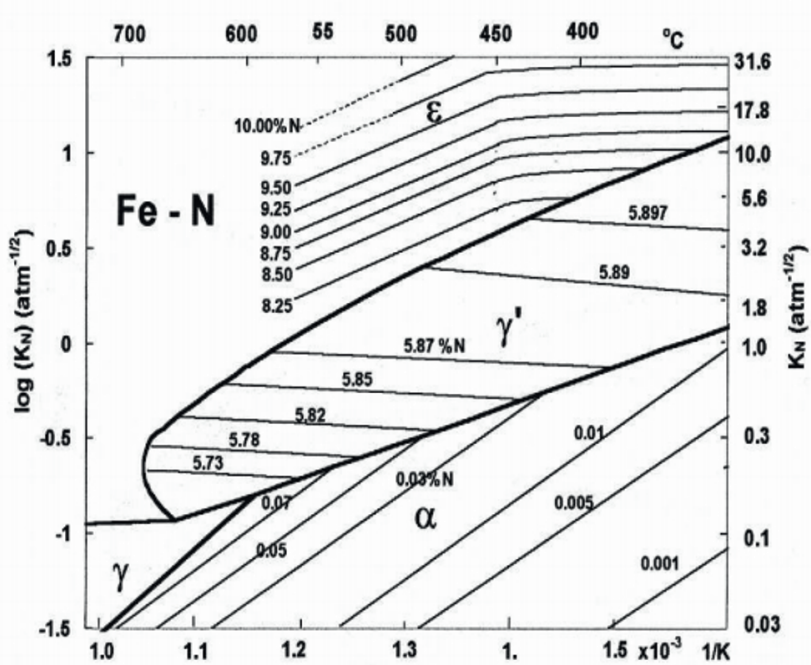

Figure 1. Diagramme d'équilibre de Lehrer actualisé par Maldzinski et al.[12]

\section{Application des deux approches thermodynamiques}

L'étude de la solubilité de l'azote dans une phase donnée repose sur l'égalité des potentiels chimiques de l'azote et du fer dans chaque phase.

Le tableau 5 donne les limites de solubilité de l'azote dans la phase $\alpha$ en équilibre avec le nitrure du fer $\gamma$ ' en utilisant les données thermodynamiques de Frisk [3]. La phase $\gamma^{\prime}$ a été modélisée comme une phase stoechiométrique, de formule chimique $\mathrm{Fe}_{4} \mathrm{~N}$ et contenant $5,89 \% \mathrm{~N}$ en poids.

Le tableau 6 contient l'évolution de la solubilité de l'azote dans les phases $\alpha$ et $\gamma$ en fonction de la température en utilisant les données thermodynamiques de la référence [3]. Ce calcul a été effectué en prenant $\mathrm{a}=3, \mathrm{c}=1$ pour la phase $\alpha, \mathrm{a}=\mathrm{c}=1$ pour la phase $\gamma$.
Tableau 5. Solubilité de l'azote dans la phase $\alpha$ en équilibre avec la phase $\gamma$,

\begin{tabular}{|c|c|}
\hline $\mathrm{T}(\mathrm{K})$ & $\begin{array}{c}\% \text { de N en poids } \\
\text { dans la phase } \alpha\end{array}$ \\
\hline 823 & 0,073 \\
\hline 833 & 0079 \\
\hline 843 & 0,085 \\
\hline 853 & 0,09 \\
\hline
\end{tabular}

Tableau 6. Solubilité de l'azote à l'interface $(\alpha / \gamma)$

\begin{tabular}{|c|c|c|}
\hline T (K) & $\begin{array}{c}\% \text { en poids dans la } \\
\text { phase } \alpha\end{array}$ & $\begin{array}{c}\% \text { en poids dans } \\
\text { la phase } \gamma\end{array}$ \\
\hline 863 & 0,098 & 2,43 \\
\hline 883 & 0,095 & 2,19 \\
\hline 900 & 0,093 & 1,99 \\
\hline 1000 & 0,065 & 0,943 \\
\hline
\end{tabular}

Nous avons comparé les résultats donnés par la théorie de Gorsky-Bragg-Williams (G-B-W) avec ceux $\mathrm{du}$ modèle des deux sous-réseaux où les phases $\gamma^{\prime}$ et $\varepsilon$ sont considérées comme des solutions solides sousrégulières.

Le tableau 7 regroupe les valeurs des concentrations en azote calculées a l'interface $\left(\alpha / \gamma^{\prime}\right)$ dans le domaine de températures allant de 823 a $853 \mathrm{~K}$. Une comparaison a été faite entre les calculs obtenus par l'utilisation du modèle de la solution solide sous-régulière [4] et ceux fournis par la théorie des isothermes d'absorption de l'azote [9-11]. La première colonne du Tableau 3 représente les valeurs des limites de solubilité inférieure (S.I) de l'azote dans la phase $\gamma^{\prime}$ tandis que la deuxième colonne donne les valeurs des limites de solubilité supérieure (S.S) en gras de l'azote dans la même phase. Il est à remarquer que la limite de solubilité de l'azote dans le nitrure du fer $\gamma^{\prime}$ varie peu avec la température. 
Tableau 7. Limites de solubilité de l'azote dans la phase $\gamma$ '. Les résultats en italique sont obtenus par le modèle de la solution sous-régulière et les résultats en gras sont ceux de la théorie des isothermes d'absorption.

\begin{tabular}{|c|c|c|c|c|}
\hline T(K) & $\begin{array}{c}\text { S.I } \\
{[4]}\end{array}$ & $\begin{array}{c}\text { S.S } \\
{[4]}\end{array}$ & $\begin{array}{c}\text { S.I } \\
{[9-11]}\end{array}$ & $\begin{array}{c}\text { S.S } \\
{[9-11]}\end{array}$ \\
\hline 823 & 0,08 & 5,74 & $\mathbf{0 , 0 7 7}$ & $\mathbf{5 , 7 4 3}$ \\
\hline 833 & 0,09 & 5,73 & $\mathbf{0 , 0 8}$ & $\mathbf{5 , 7 4 5}$ \\
\hline 843 & 0,09 & 5,71 & $\mathbf{0 , 0 8 7}$ & $\mathbf{5 , 7 4}$ \\
\hline 853 & 0,09 & 5,86 & $\mathbf{0 , 0 9}$ & $\mathbf{5 , 7 2 1}$ \\
\hline
\end{tabular}

Le Tableau 8 donne également les valeurs des concentrations en azote calculées a l'interface $\left(\gamma^{\prime} / \varepsilon\right)$ dans le domaine de températures allant de 823 à $853 \mathrm{~K}$. La première colonne du Tableau 8 représente les valeurs des limites de solubilité inférieure (S.I) de l'azote dans la phase $\varepsilon$ et la deuxième colonne est relative aux valeurs des limites de solubilité supérieure (S.S) de l'azote dans la même phase. Un écart remarquable est manifeste pour la solubilité supérieure de l'azote dans la phase $\varepsilon$ où les valeurs données par Du [4] sont supérieures à celles obtenues par la théorie des isothermes d'absorption de l'azote.

Tableau 8. Limites de solubilité de l'azote dans la phase $\varepsilon$.

Les résultats en italique sont obtenus par le modèle de la solution sous- régulière et les résultats en gras sont ceux de la théorie des isothermes d'absorption de l'azote.

\begin{tabular}{|c|c|c|c|c|}
\hline T (K) & $\begin{array}{c}\text { S.I } \\
{[4]}\end{array}$ & $\begin{array}{c}\text { S.S } \\
{[4]}\end{array}$ & $\begin{array}{c}\text { S.I } \\
{[9-11]}\end{array}$ & $\begin{array}{c}\text { S.S } \\
{[9-11]}\end{array}$ \\
\hline 823 & 5,86 & 7,33 & $\mathbf{5 , 8 7 7}$ & $\mathbf{7 , 7 9 4}$ \\
\hline 833 & 5,86 & 7,25 & $\mathbf{5 , 8 7 6}$ & $\mathbf{7 , 6 6 6}$ \\
\hline 843 & 5,86 & 7,16 & $\mathbf{5 , 8 7 5}$ & $\mathbf{7 , 5 8 3}$ \\
\hline 853 & 5,85 & 7,07 & $\mathbf{5 , 8 7 5}$ & $\mathbf{7 , 5 6 2}$ \\
\hline
\end{tabular}

Cet écart, entre les résultats calculés, est dû à l'instabilité des nitrures du fer par rapport à l'azote moléculaire. Les résultats de la théorie des isothermes d'absorption de l'azote tiennent compte de l'arrangement des atomes de l'azote dans le deuxième sous-réseau des phases $\gamma^{\prime}$ et $\varepsilon$.

La figure 2 donne d'ailleurs un tracé partiel du diagramme binaire des phases $\mathrm{Fe}-\mathrm{N}$ qui montre un domaine de composition très étroit pour la phase $\gamma^{\prime}$ contrairement aux travaux des auteurs [1-2], où elle a été définie comme une phase stœchiométrique de composition fixe.

\section{Conclusion}

Dans ce travail, une étude thermodynamique des phases $\gamma^{\prime}$ et $\varepsilon$ a été effectuée en utilisant deux approches de calcul des limites de solubilité de l'azote dans ces deux phases. La comparaison des résultats du modèle de la solution régulière ou sous- régulière avec ceux trouvés par la théorie des isothermes d'absorption de l'azote a permis de montrer la conformité des résultats de cette dernière avec les données expérimentales car elle considère un équilibre thermodynamique entre les phases nitrures et le mélange gazeux $\mathrm{NH}_{3}-\mathrm{H}_{2}$.

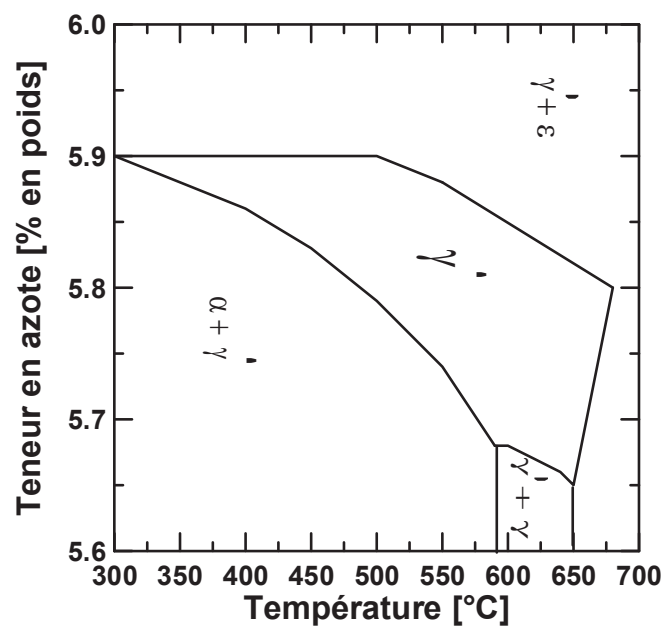

Figure 2. Diagramme d'équilibre partiel Fe-N tracé par la théorie des isothermes d'absorption de l'azote.

\section{Références}

1. M. Hillert, L. I. Staffansson, Acta Chem. Scan. A. 24, 3618 (1970)

2. J. Agren, Metall. and Mater. Trans. 10 A, 1847, (1979)

3. K. Frisk, Calphad, 15, 76 (1991)

4. H. Du, J. Phase Equilibria. 14, 682 (1993)

5. J. Kunze, Steel Res., 57, 361 (1986)

6. M.K.Lei, X.J. Wang, Chin. Phys. Lett., 19, 1721 (2002)

7. A.F. Guillermet, H.Du, Z. Metallkde, 85, 154 (1994)

8. G. Inden, Calphad, 4, 111 (1976)

9. B.J. Kooi, M.A.J. Somers, E.J. Mittemeijer, Metall. and Mater. Trans., 27 A, 1055 (1996)

10. B.J. Kooi, M.A.J. Somers, E.J. Mittemeijer, Metall. and Mater. Trans., 27 A, 1063 (1996)

11. M.A.J. Somers, B.J. Kooi, L. Maldzinski, E.J. Mittemeijer,A. A. Van Der Horst, A. M. Van Der Kraan, N.M. Van Der Pers, Acta Mater., 45, 2013 (1997)

12. L.Maldzinski,W.Liliental,G.Tymowski,J.Tacikowski ,Surf. Eng, 15, 377 (1999) 